\title{
Challenges in the Development of Health System Performance Dashboard for Malaysia
}

Siti Ramizah Ramli', Zainanda Zainal', Jamaiyah Haniff', Anis Farhana Badrul Hisham', Dhesi Baha Raja a/l Selva Raja²

${ }^{1}$ Malaysian Healthcare Performance Unit, Research Policy and Planning Division, National Institute of Health, Ministry of Health Malaysia, W.P. Kuala Lumpur, 50586, Malaysia

${ }^{2}$ Minister's office, Ministry of Health Malaysia, W.P. Putrajaya, 62590, Malaysia

\begin{abstract}
Introduction: Traditional static reports of performance monitoring in healthcare sector is inconsistent, incomparable and time-consuming. Incapable of reflecting the whole-of-system or effectively support decision making, that a digitalized interactive tool for measuring, monitoring, and managing performance is mandatory. Our aims are: (1) Enculturation of integrative healthcare performance; (2) Addressing challenges to development of the Health System Performance Dashboard (HSPD) for Malaysia; and (3) Invitation for smart partnership.
\end{abstract}

Methods: There are six steps for a dashboard implementation and development from Dashboard Development and Deployment: A Methodology for Success by Noetix Corporation. This proof of concept project has approaching the end of second stage; building a prototype. We discussed on the challenges that we encountered until the development of the prototype and the proposed recommendations on each challenges.

Results: The first step in developing a dashboard is planning. This involves team member's identification and roles, determining project objectives and scope, setting a timeline and budget, and defining strategic Key Performance Indicators (KPIs). Second step is the requirements gathering by interviewing stakeholders, setting expectations, explore functionality, define data types and build a prototype.

Conclusion: Digitalization and HSPD are warranted to assist decision-makers to prioritize on a larger scale. A key success factor to realization of HSPD is an anchored data scientist team. This calls for an urgent and important smart partnership with the experts. A.I. expertise is needed for continuous diagnostic, predictive and prescriptive analytics of the massive healthcare data.

Acknowledgement: Director General of Health, Deputy Director General of Health (Research and Technical Support), Director of Research Policy and Planning Division, National Institute of Health

Keywords: Performance dashboard, Health system, Data scientist team 